\title{
The transcriptional repressor Brinker antagonizes Wingless signaling
}

\author{
Elisabeth Saller, Ann Kelley, and Mariann Bienz ${ }^{1}$ \\ Medical Research Council Laboratory of Molecular Biology, Cambridge, CB2 2QH, UK
}

In the embryonic midgut of Drosophila, Wingless (Wg) signaling elicits threshold-specific transcriptional response, that is, low-signaling levels activate target genes, whereas high-signaling levels repress them.

Wg-mediated repression of the $H O X$ gene Ultrabithorax $(U b x)$ is conferred by a response sequence within the Ubx B midgut enhancer, called WRS-R. It further depends on the Teashirt (Tsh) repressor, which acts through the WRS-R without binding to it. Here, we show that Wg-mediated repression of $U b x$ B depends on Brinker, which binds to the WRS-R. Furthermore, Brinker blocks transcriptional activation by ubiquitous Wg signaling. Brinker binds to Tsh in vitro, recruits Tsh to the WRS-R, and we find mutual physical interactions between Brinker, Tsh, and the corepressor dCtBP. This suggests that the three proteins may form a ternary repressor complex at the WRS-R to quench the activity of the nearby-bound dTCF/Armadillo transcription complex. Finally, brinker and $t$ sh produce similar mutant phenotypes in the ventral epidermis, and double mutants mimic overactive Wg signaling in this tissue. This suggests that Brinker may have a widespread function in antagonizing Wg signaling.

[Key Words: Drosophila; Brinker; transcriptional repression; Teashirt; dCtBP; Wingless]

Received March 6, 2002; revised version accepted May 14, 2002.

Wingless $(\mathrm{Wg})$ signaling is essential at different stages during Drosophila development. In the early embryo, wg is expressed in segmental stripes in the epidermis, and together with engrailed determines the parasegmental borders (Perrimon 1994). Later, during embryogenesis, $w g$ has a critical function during endoderm induction: $w g$ is expressed in a single parasegment (ps) in the midgut, in which it controls the expression of two HOX genes, Ultrabithorax $(U b x)$ and labial, as well as the formation of the middle gut constriction, which separates the anterior and the posterior midgut (Bienz 1994). In imaginal discs, Wg signaling controls multiple processes, including the specification of ventral cell fates in the leg disc (Struhl and Basler 1993), the dorso-ventral compartment boundary, the prospective margin in the wing disc (Klein 2001), and the formation of head cuticle in the eye disc (Treisman and Rubin 1995; Royet and Finkelstein 1996).

Armadillo is a key effector of the Wg pathway (Cavallo et al. 1997). It is stabilized by Wg signaling and, consequently, translocates into the nucleus, in which it activates the transcription of $\mathrm{Wg}$ target genes by binding to dTCF (Brunner et al. 1997; Riese et al. 1997; van de Wetering et al. 1997). dTCF belongs to the TCF/LEF family of sequence-specific high mobility group (HMG) proteins

${ }^{1}$ Corresponding author.

E-MAIL mb2@mrc-lmb.cam.ac.uk; FAX 44-1-223-412142.

Article and publication are at http://www.genesdev.org/cgi/doi/10.1101/ gad.230002. that are thought to be architectural factors mediating assembly of multiprotein enhancer complexes (Grosschedl et al. 1994). In the absence of Wg signaling, dTCF actively represses $\mathrm{Wg}$ target genes by binding to the corepressor Groucho (Cavallo et al. 1998) and the CREBbinding protein (dCBP), which can acetylate the Armadillo-binding domain of dTCF (Waltzer and Bienz 1998). Thus, Wg signaling converts dTCF from a transcriptional repressor to an activator (Bienz 1998).

A number of $\mathrm{Wg}$ target genes have been identified whose expression is stimulated directly by dTCF/Armadillo. These include $U b x$ (Riese et al. 1997), dpp (Yang et al. 2000), and stripe (Piepenburg et al. 2000), each of which contains Wg-responsive enhancers with binding sites for dTCF. Other examples are teashirt (tsh) (Mathies et al. 1994), engrailed (Hooper 1994), Dfrizzled2 (Cadigan et al. 1998), and shavenbaby (Payre et al. 1999), but it has not been shown whether these genes are controlled directly by dTCF. Furthermore, Wg signaling can also repress target genes, for example the HOX genes $U b x$ and labial in the embryonic midgut (Hoppler and Bienz 1995; Yu et al. 1998). Similarly, Wg represses its own expression in the midgut (Yu et al. 1998) and in the wing imaginal disc along the margin (Rulifson et al. 1996). $d p p$ is antagonized by Wg in multiple embryonic and larval tissues (Ma and Moses 1995; Treisman and Rubin 1995; Brook and Cohen 1996; Jiang and Struhl 1996). Notably, in some tissues, Wg signaling acts at multiple threshold levels to control the expression of its target genes, for example, in the embryonic midgut. In 
this issue, $U b x$ and labial are stimulated by low $\mathrm{Wg}$ levels and repressed by high Wg levels (Bienz 1997). Wg also acts at multiple thresholds in the wing (Zecca et al. 1996; Neumann and Cohen 1997) and in the leg imaginal discs (Lecuit and Cohen 1997).

Transcriptional repression mediated by Wg is not well understood. Perhaps the best-studied model is the midgut enhancer of $U b x$, called $U b x \mathrm{~B}$ (Thüringer et al. 1993), which is repressed by high levels of Wg signaling in the posterior embryonic midgut (Yu et al. 1998; Fig. 1A). This repression is conferred by the WRS-R (Yu et al. 1998), a sequence that is distinct from the WRS, that is, the dTCF-binding site that confers Wg-mediated stimulation of this enhancer (Riese et al. 1997; Fig. 1B). The WRS-R coincides with the DRS, a tandem of binding sites for the Dpp effector Mad that mediates transcriptional stimulation by Dpp signaling (Kim et al. 1997; Szüts et al. 1998). Furthermore, it has been shown that Tsh acts through the WRS-R to repress $U b x$ B in response to high Wg levels (Waltzer et al. 2001). However, Tsh does not bind to the WRS-R directly (Waltzer et al. 2001), so the DNA-binding protein conferring the Wgmediated repression remained elusive.

Brinker was initially discovered as an antagonist of Dpp signaling (Campbell and Tomlinson 1999; Jazwinska et al. 1999a; Minami et al. 1999). It is a sequencespecific DNA-binding protein with a distantly related homeodomain (Sivasankaran et al. 2000; Rushlow et al. 2001; Zhang et al. 2001) and functions as a transcriptional repressor (Sivasankaran et al. 2000; Hasson et al.

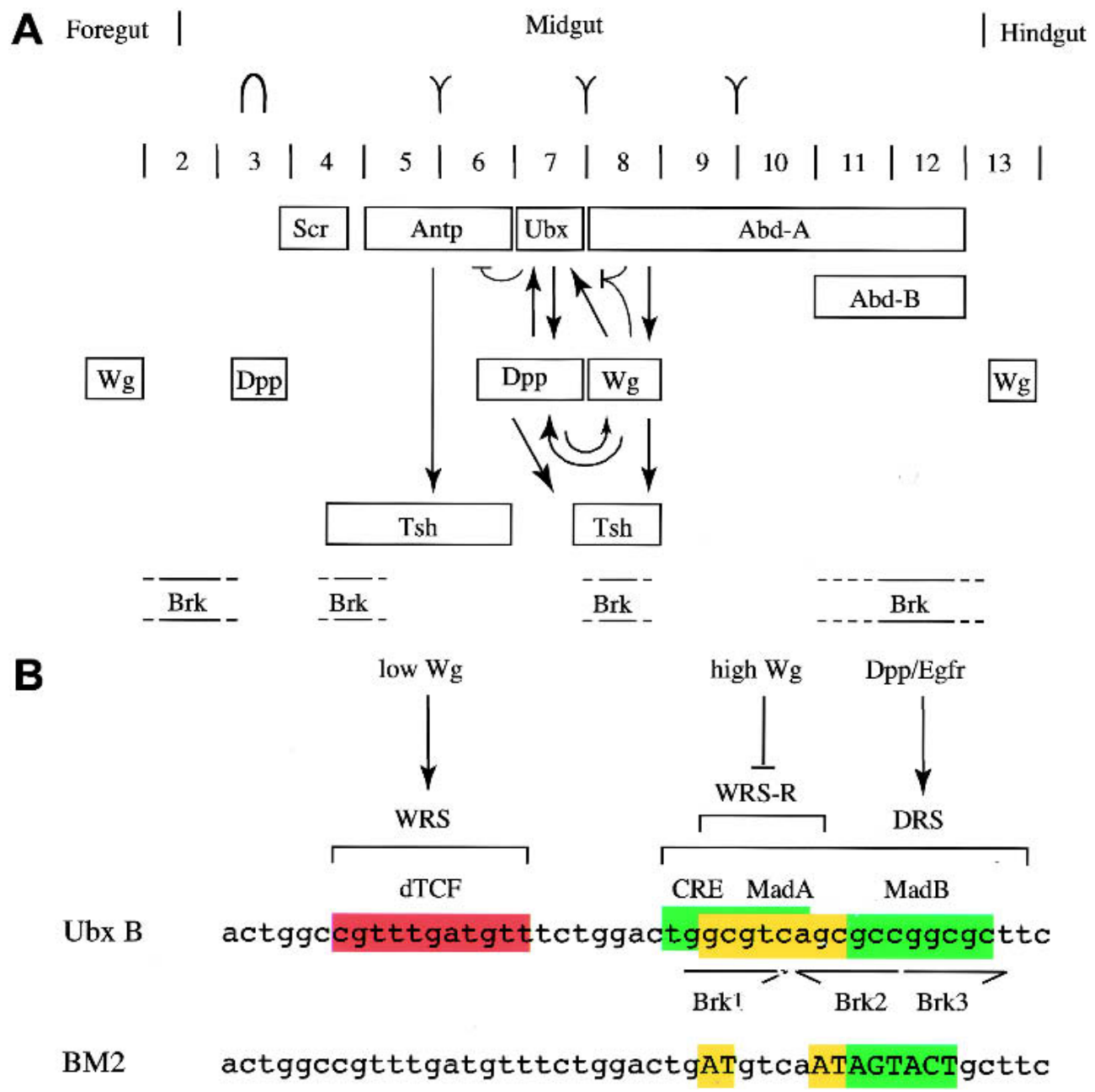

Figure 1. Gene expression in the midgut and signal-responsive sequences in $U b x$ B. (A) Expression of HOX proteins (top), Wg and Dpp in the midgut mesoderm (Bienz 1996) in relation to Tsh expression and estimated domains of brk expression (Jazwinska et al. 1999b; E. Saller and M. Bienz, unpubl.). Expression of $t s h$ in ps8 requires $w g$ and $d p p$ (Mathies et al. 1994). Midgut limits, positions of gastric caeca (in ps3) and of the gut constrictions are indicated above parasegments (ps); note that the middle gut constriction bissects the midgut into anterior and posterior. Critical regulatory interactions between the genes in the middle midgut are shown (arrows, stimulatory; barred line, repressive). (B, top) Signal-responsive sequences within the Ubx B midgut enhancer, with the WRS, DRS, and WRS-R indicated. These include binding sites for the Wg effector dTCF, the Dpp effector Mad, and the cAMP response element (CRE)-like sequence. Note that the three Brinker-binding sites coincide with the Mad sites (Saller and Bienz 2001). (Bottom) Sequence of the BM2 enhancer with mutations in the Mad/Brinker-binding sites. 
2001; Kirkpatrick et al. 2001; Rushlow et al. 2001; Zhang et al. 2001). Recently, we have discovered that Brinker binds to the Mad-binding sites within $U b x$ B to antagonize Dpp-mediated stimulation of this enhancer (Saller and Bienz 2001). Because these overlap the WRS-R (see above), we asked whether Brinker might be involved in Wg-mediated repression. This is the case. Here, we show that brinker is required for repression of $U b x$ and $w g$ by high levels of $\mathrm{Wg}$ signaling in the embryonic midgut. Furthermore, Brinker competes efficiently with Wg signaling, blocking Wg-mediated stimulation of $U b x$ B. We provide evidence that Brinker can recruit Tsh to the WRS-R to form a repressor complex, and that the two proteins can recruit the corepressor $\mathrm{dCtBP}$. This suggests a mechanism by which Brinker can block dTCF/Armadillo-mediated stimulation of Wg target genes. Finally, we show that Brinker also antagonizes $w g$ in the ventral epidermis of Drosophila embryos.

\section{Results}

brinker is required for Wg-mediated repression of Ubx and wg

In wild-type embryos, $U b x \mathrm{~B}$ directs $\beta$-galactosidase (lacZ) expression in the middle midgut, between the anterior and the posterior midgut constriction (ps6-9) (Fig. 2A). In brinker mutants, $U b x \mathrm{~B}$ is derepressed at both ends of the midgut, approximately in ps2 and ps12 (Saller and Bienz 2001; arrowheads in Fig. 2B). If Wg is overexpressed throughout the midgut, lac $Z$ staining is strong anterior to the middle gut constriction (i.e., to the left of the vertical bar in Fig. 2C), but is much reduced posterior to this constriction (Fig. 2C, open triangle; $\mathrm{Yu}$ et al. 1998). The stimulation in the anterior midgut is partly due to the overexpressed $\mathrm{Wg}$, and partly to endogenous
Dpp, which is ectopically activated throughout this region by constitutive Wg signaling (Yu et al. 1996). The lack of staining posterior to the middle gut constriction reflects repression of $U b x \mathrm{~B}$ by $\mathrm{Wg}$ signaling, which reaches particularly high levels near the Wg source (in ps8, indicated by asterisks in Fig. 2; Yu et al. 1998). However, if $\mathrm{Wg}$ is overexpressed in brinker mutant embryos, lacZ staining is evenly strong throughout the midgut, from ps2 to ps12 (Fig. 2D). Thus, brinker is required in the posterior midgut for the repression of $U b x \mathrm{~B}$ in response to high Wg levels.

We asked whether brinker mutation affects $U b x$ itself. $U b x$ is expressed in ps 7 of the midgut, with a sharp posterior expression limit coinciding with the middle gut constriction (Bienz and Tremml 1988). Although $U b x$ is derepressed in the posteriormost region of the midgut in brinker mutants, we could not detect any $U b x$ derepression into ps8 (Saller and Bienz 2001), probably because $U b x$ is repressed in this region by the $H O X$ gene $a b$ dominal-A (presumably independently of Brinker; Bienz and Tremml 1988). We thus used an extended $U b x$ enhancer, called RP9, which faithfully mimics Ubx expression in the midgut, but which is less efficiently repressed by abdominal- $A$ than $U b x$ itself (Thüringer and Bienz 1993). Like $U b x$ itself, RP9 directs $l a c Z$ expression in ps7 of the visceral mesoderm (Fig. 2E) in response to Dpp and low levels of $\mathrm{Wg}$ signaling, but is repressed posterior to the middle gut constriction in response to high $\mathrm{Wg}$ levels (in contrast to $U b x \mathrm{~B}$, which has lost much of its responsiveness to high Wg levels, and thus mediates broader expression; Yu et al. 1998). In brinker mutant embryos, RP9-mediated lacZ staining extends clearly beyond the middle gut constriction and is strong in ps8 (Fig. 2F; Saller and Bienz 2001). Furthermore, in brinker mutants, $\mathrm{RP9}$ responds to ubiquitous $\mathrm{Wg}$ in the posterior midgut by conferring lacZ staining in this region (arrow-

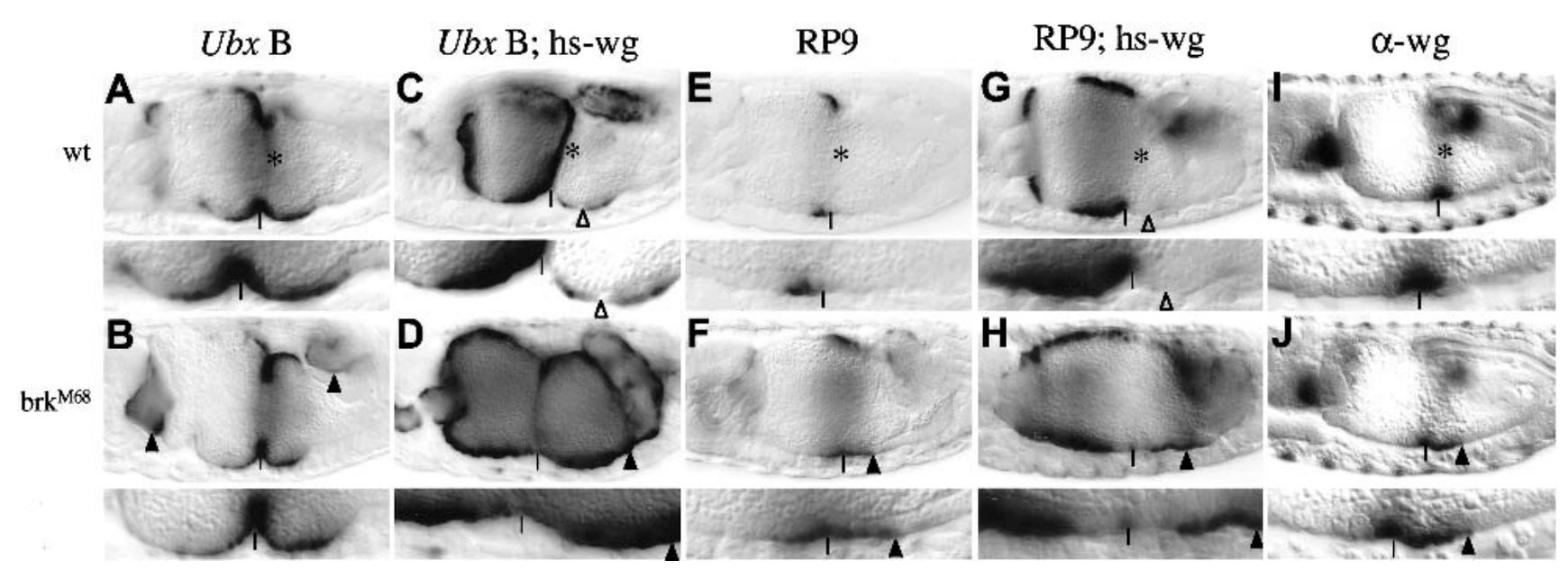

Figure 2. brinker is required for Wingless-mediated repression of $U b x$ and $w g$. Side views of 12-to-14-hour-old embryos, wild-type or brk mutant, bearing $U b x$ reporter genes and hs-wg as indicated, stained with antibody against lacZ $(A-H)$ or Wg $(I, J)$; $(b o t t o m)$ an enlarged section is shown that spans the relevant gut region (ps6-ps9). $\left({ }^{\star}\right)$ Wingless sources in the wild type; $(\triangle)$ point to transcriptional repression due to high $\mathrm{Wg}$ levels, arrowheads to stimulation and derepression in response to ectopic Wg. Note the absence of Wg-mediated repression posterior to the middle gut constrictions in brk mutants. These constrictions are indicated by vertical lines in this and all subsequent figures; anterior to the left, dorsal up. 
head in Fig. $2 \mathrm{H}$ ), although there is no trace of RP9-mediated staining in this region in a wild-type embryo that expresses ubiquitous $\mathrm{Wg}$ (open triangle in Fig. 2G). These results indicate that brinker is required for repression of RP9-and by extrapolation of Ubx-posterior to the middle gut constriction, near the $\mathrm{Wg}$ source.

Next we asked whether brinker also controls $w g$, which, at late embryonic stages, autorepresses itself in ps8 (Yu et al. 1998). In wild-type embryos, wg is initially expressed in ps8 (Bienz et al. 1988), but subsequently, when the middle gut constriction forms, shifts slightly toward anterior, so it is expressed at this stage in a band of 6-8 cells wide spanning this constriction (Fig. 2I). However, in brinker mutant embryos, there is a clear posterior expansion of $\mathrm{Wg}$ staining that is now detectable in a band of $\sim 8-12$ cells wide (Fig. 2J, arrowhead). Thus, brinker is required for autorepression of $w g$ in this region of the midgut.

\section{Brinker repressor blocks Wg-induced stimulation of target genes}

Our results suggest that the Brinker repressor may be able to overcome $\mathrm{Wg}$-mediated target gene activation in the middle midgut. To test this hypothesis, we monitored the activity of $U b x \mathrm{~B}$ after simultaneous overexpression of Brinker and Wg in the mesoderm. As mentioned above, $\mathrm{Wg}$ overexpression alone leads to strong staining in the anterior part of the midgut (ps3-ps7) and substantial reduction of staining posterior to the middle gut constriction (Figs. 2A,C and 3A,C). However, ectopic

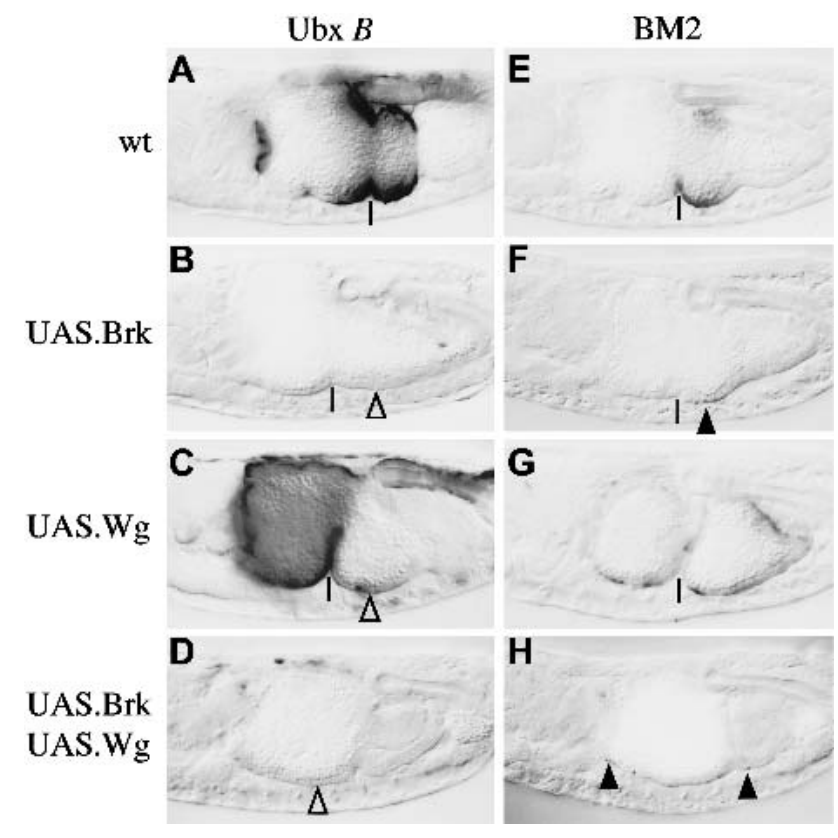

Figure 3. Brinker blocks Wg-induced transcriptional stimulation by targeting the WRS-R. Side view of $\sim 14$-hour-old embryos bearing $U b x$ B or BM2 as indicated, stained with antibody against lacZ. $(\triangle)$ Indicates lack of lacZ staining due to ectopic $\mathrm{Wg}$ or Brinker; residual staining is indicated by arrowheads.
Brinker repressed $U b x$ B virtually throughout the midgut, whether or not Wg was present (Fig. 3B,D). This shows that Brinker can repress a Wg-responsive enhancer even in the presence of strong Wg stimulation. Evidently, the Brinker repressor is dominant over the stimulatory dTCF/Armadillo transcription complex.

\section{Brinker targets the WRS-R to repress $\mathrm{Ubx} B$ in response to $W g$}

A mutant $U b x$ B enhancer that lacks functional Madbinding sites (BM2) directs weak lacZ expression posterior to the middle gut constriction (in ps8 and ps9), which reflects its responsiveness to Wg stimulation but not to Dpp (Szüts et al. 1998; Fig. 3E). As expected, BM2 is much less responsive to overexpressed Brinker than the wild-type enhancer (Saller and Bienz 2001), although we noted earlier that there is still a residual Brinker response of BM2 (as indicated by the slight difference in lacZ staining between Fig. 3F and E). This residual response could be due to a fortuitous Brinker-binding site elsewhere in the plasmid (Saller and Bienz 2001).

Ectopic Wg causes an expansion of BM2-mediated lac $Z$ staining in cells scattered throughout the midgut (including the posterior midgut, in which $\mathrm{Wg}$ represses Ubx B; Fig. 3G). This confirms that this mutant enhancer can be stimulated, but no longer repressed, by Wg signaling (Yu et al. 1998). Simultaneous overexpression of Brinker and $\mathrm{Wg}$ also allows activity of BM2 in individual cells throughout the midgut (Fig. 3H). This lacZstaining pattern is similar to that due to ectopic $\mathrm{Wg}$ alone (Fig. 3G), although, again, there is less lacZ staining in the presence compared with the absence of ubiquitous Brinker, indicating a residual Briner response of BM2 also under these conditions. Nevertheless, these experiments indicate that Brinker depends on the WRS-R sequence to fully antagonize Wg-mediated stimulation of $U b x$ B.

\section{Brinker recruits Tsh and dCtBP to the WRS-R}

We asked whether Brinker might recruit Tsh to the WRS-R. We thus expressed Tsh in bacteria as a GST fusion protein and tested its binding to radioactively labeled in vitro-translated Brinker by pull-down assays. This revealed binding between the two proteins in vitro (Fig. 4A, lanes 1-3). This was confirmed by the conversebinding experiment based on GST-Brinker and radioactively labeled Tsh (Fig. 4A, lanes 4-6).

Next, we performed gel-shift assays to test whether Tsh can bind to Brinker when the latter is bound to the WRS-R. We have shown previously that the amino-terminal homeodomain of Brinker (amino acids 44-99) binds to the Mad-binding sequence within $U b x \mathrm{~B}$, whereas a carboxy-terminal fragment of Brinker lacking this domain does not (Saller and Bienz 2001). We now find that GST-Tsh produces a super-shift if added to fulllength Brinker (Fig. 4B, lanes 10 and 11) or to an aminoterminal fragment of Brinker (spanning amino acids 
Saller et al.
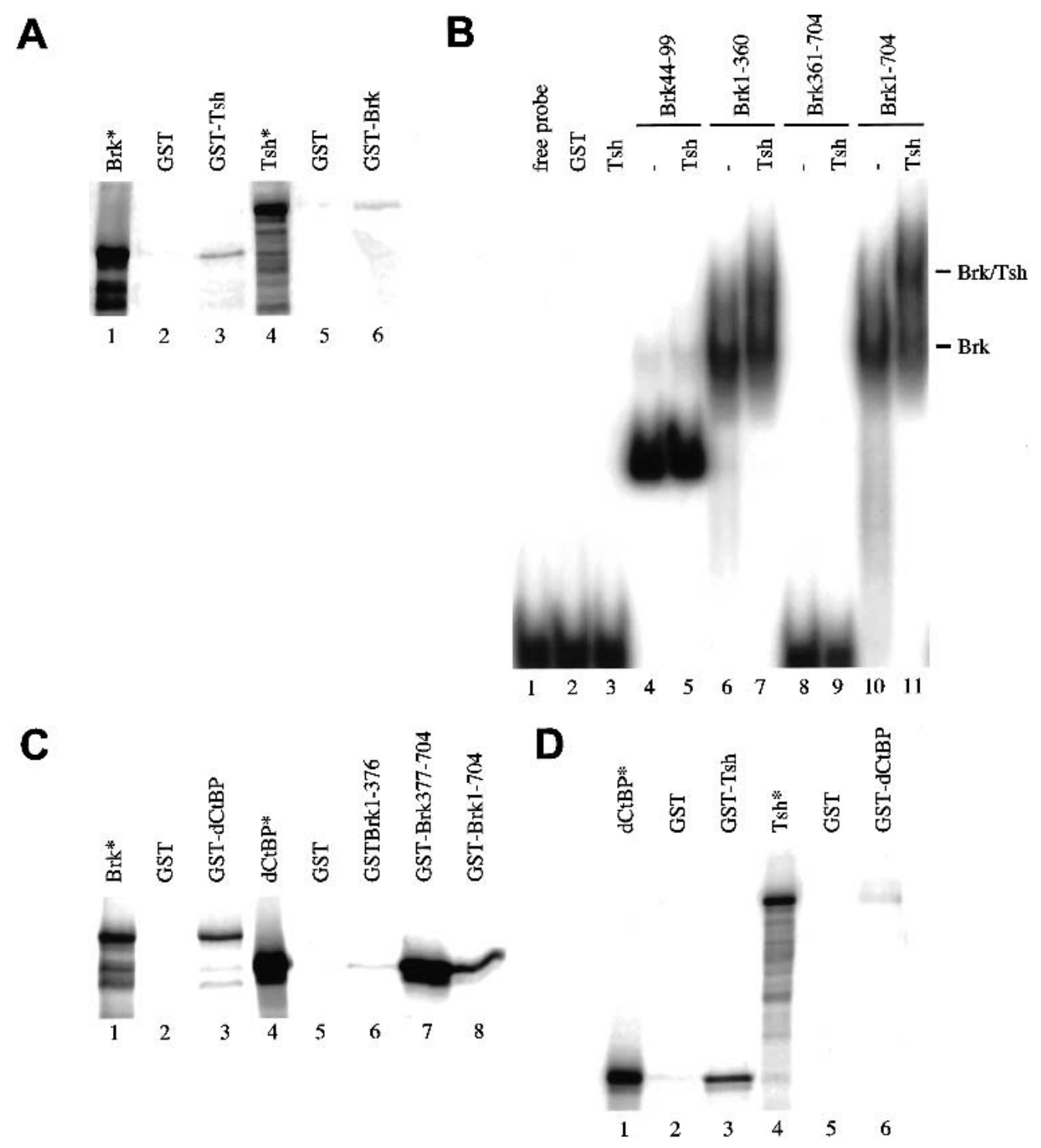

Figure 4. Direct binding between Brinker, Tsh, and dCtBP. $(A, C, D)$ In vitro pull-down assays with equimolar amounts of GST fusion protein and in vitro-translated radioactively labeled protein as indicated; $10 \%$ of the total reaction was loaded in the input lane $\left({ }^{\star}\right) .(B)$ Gel-shift assays with GST fusion proteins as indicated at top $(1 \mu \mathrm{g}$ per assay for Brinker-GST or fragments thereof; $15 \mu \mathrm{g}$ per assay for Tsh-GST). The positions of the Brinker/DNA complex and of the ternary Tsh/Brinker/DNA complex are indicated at right.

1-360; Fig. 4B, lanes 6 and 7), but fails to produce a supershift if added to a minimal Brinker fragment spanning the homeodomain (Fig. 4B, lanes 4 and 5). This indicates that Brinker can recruit Tsh to the WRS-R.

Brinker and Tsh both contain a motif (P-DLS-K) known to interact with Drosophila CtBP (dCtBP) (Nibu et al. 1998b). Human CtBP was first identified as a protein that binds to the carboxyl terminus of the adenovirus E1a oncoprotein and functions as a tumor suppressor (Boyd et al. 1993). Furthermore, CtBP proteins are transcriptional corepressors (Turner and Crossley 1998; Criqui-Filipe et al. 1999). dCtBP was isolated in a twohybrid screen for proteins that bind to the transcriptional repressor Hairy (Poortinga et al. 1998; Zhang and Levine 1999; Phippen et al. 2000). It mediates transcriptional repression by the short-range repressors Knirps, Krüppel,
Snail, and Giant in the Drosophila embryo (Nibu et al. 1998a; Nibu and Levine 2001). We thus tested in vitro binding between Brinker and $\mathrm{dCtBP}$, and between Tsh and dCtBP.

In vitro-translated Brinker binds to bacterially expressed GST-dCtBP in pull-down assays (Fig. 4C, lane 3). As expected, an amino-terminal fragment of Brinker spanning amino acids 1-376 fails to bind in vitro-translated dCtBP (Fig. 4C, lane 6). However, a carboxy-terminal fragment of Brinker spanning amino acids 377-704, and to a lesser extent, full-length GST-Brinker, bind to dCtBP (Fig. 4C, lanes 7 and 8). Further pull-down assays show direct binding between dCtBP and Tsh (Fig. 4D). These results suggest that Brinker and Tsh may be able to recruit the corepressor $\mathrm{dCtPB}$, individually or together. 
The repressor activity of Tsh in the midgut depends on brinker

We tested whether Brinker and Tsh interdepend on each other in the transcriptional repression of $U b x$ B. tsh mutant embryos are easily recognized by their lack of the anterior and middle gut constrictions (Mathies et al. 1994), but the staining pattern of $U b x$ B in tsh mutant embryos is the same as the pattern seen in wild-type embryos (Fig. 5A). Ectopic Brinker repressed $U b x \mathrm{~B}$ throughout the midgut, regardless of whether Tsh was present or not (Fig. 5B,C). Thus, Brinker can repress the $U b x$ B enhancer in the absence of Tsh.

We then asked whether Tsh can repress transcription in the absence of Brinker. Overexpression of Tsh throughout the mesoderm causes repression of $U b x \mathrm{~B}$ anterior to the middle gut constriction (in ps6 and ps7), but not in ps8, in which the levels of endogenous Wg and Tsh are high (Waltzer et al. 2001; Fig. 5E). However, Tsh cannot repress $U b x \mathrm{~B}$ in brinker mutant embryos; the lacZ staining pattern is the same in these mutants, whether or not they also overexpress Tsh (Fig. 5, cf. F with D). Thus, Tsh depends on brinker to repress $U b x \mathrm{~B}$ in the midgut.

\section{Brinker and Tsh antagonize Wg signaling in the ventral embryonic epidermis}

brinker was identified originally in a screen for mutants that affect Dpp signaling in the ventral epidermis (Jazwinska et al. 1999a). These authors described the brinker
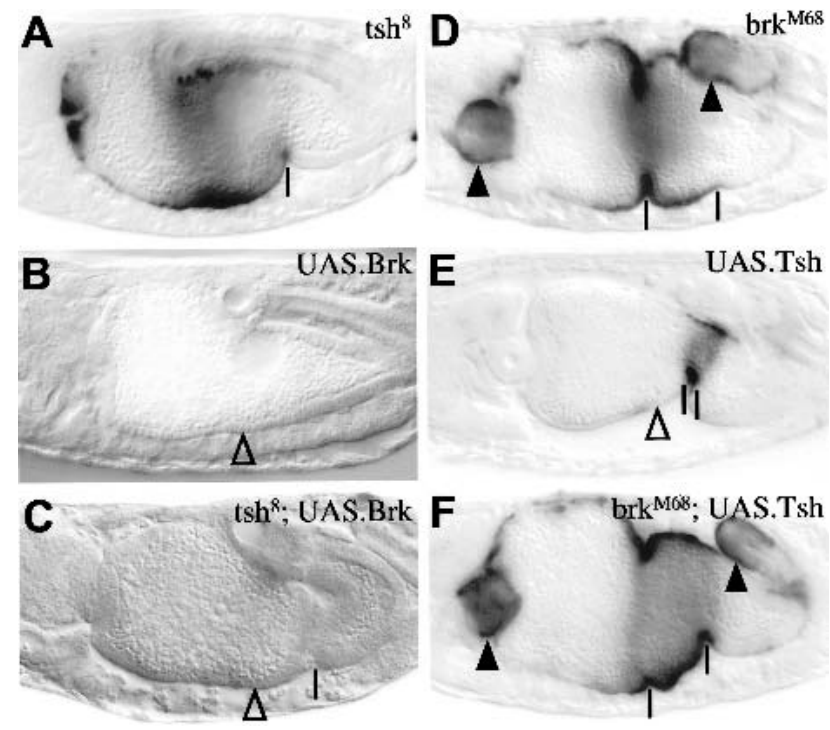

Figure 5. Tsh-mediated repression of $U b x$ B depends on brinker. Side view of $\sim 14$-hour-old mutant embryos as indicated, bearing $U b x \mathrm{~B}$ and stained with antibody against lacZ. $(\triangle)$ Indicates lack of lacZ staining due to ectopic Brinker or Teashirt; derepression of staining is indicated by arrowheads. Note the absence of the anterior and middle gut constrictions in $t s h$ mutants (Mathies et al. 1994) and the absence of the anterior constriction in UAS.Tsh embryos (Waltzer et al. 2001).
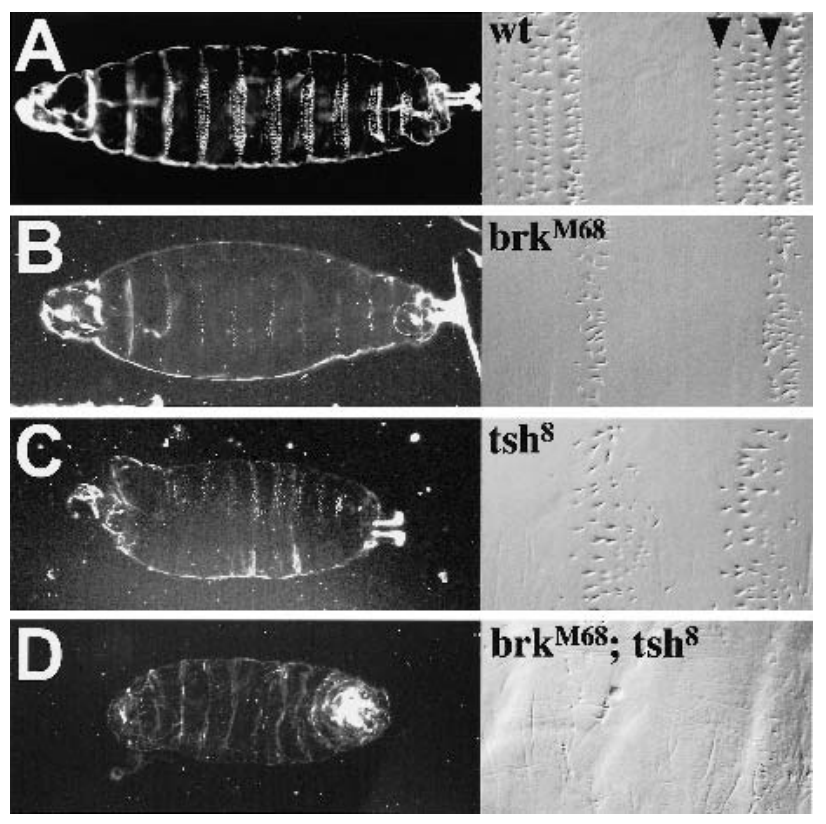

Figure 6. brk and tsh antagonize $\mathrm{Wg}$ signaling in the ventral embryonic epidermis. Ventral views of larval cuticles, wild type or mutant as indicated, viewed by dark field illumination (left) or by Nomarski optics at high magnification (right; abdominal denticle belts shown). Arrowheads point to denticle rows 1 and 4 .

mutant phenotype of the larval cuticle, and interpreted this to reflect an expansion of the dorsal epidermis at the expense of the ventral epidermis-consistent with an antagonistic effect of brinker on dorsal Dpp signaling.

However, when we re-examined the cuticles of brk ${ }^{M 68}$ mutants (the allele described by Jazwinska et al. 1999a), we found that these primarily show a segment polarity phenotype in the ventral epidermis. Wild-type cuticles show segmental stretches of naked cuticle alternating with denticle belts (Fig. 6A, left). In the abdomen, the latter consist of six characteristic rows of denticles, small denticles with hooked ends and flat bases in rows 1-4, large tapered denticles that are less hooked in row 5 , and tiny tapered denticles in row 6 . The denticles of each row have a characteristic polarity with denticles in rows 1 and 4 pointing toward the anterior, whereas the others point toward the posterior (Fig. 6A, right). brinker mutant larvae show narrowed denticle belts (Fig. 6B, left) of about three disordered rows of tapered denticles, all of which point toward the posterior, usually one row of large denticles followed by two rows of tiny denticles (Fig. 6B, right). Another brinker allele $\left(b r k^{F 124}\right.$; Lammel et al. 2000), which is less strong than $b r k^{M 68}$ shows essentially the same mutant phenotype as brk ${ }^{M 68}$, except that the residual denticle belts are slightly broader (data not shown). Therefore, brinker loss clearly causes a major change in the structure and organization of each individual segment in the larval abdomen. Interestingly, this phenotype is also caused by ectopic Wg signaling, which antagonizes epidermal growth factor receptor (EGFR) signaling in the embryonic epidermis (O'Keefe et al. 1997; Szüts et al. 1997). 
Notably, tsh mutant embryos also retain residual denticle belts with only tapered denticles of the row 5 and 6 type (Gallet et al. 2000; Fig. 6C). The phenotypic similarity between brinker and tsh mutant embryos in the ventral epidermis suggests that both proteins may be involved in the same pathway. To test this hypothesis, we constructed a double-mutant brinker tsh strain and examined their ventral cuticles. These clearly look more severe than either single mutant; they do not show any residual denticle belts (Fig. 6D, left), and only single tiny denticles can be observed occasionally scattered in the posterior abdomen (Fig. 6D, right). In other words, these embryos essentially show the naked cuticle phenotype that results from ubiquitous $\mathrm{Wg}$ expression in the embryonic epidermis (Perrimon 1994). This indicates that Brinker and Tsh synergize to promote the formation of ventral denticle belts. In support of this, both brinker and tsh single mutants show loss of segmental rhomboid expression in the trunk of the embryonic epidermis (data not shown), implying that EGFR signaling is not activated in these mutants (O'Keefe et al. 1997; Szüts et al. 1997). As a consequence, shavenbaby fails to be activated (data not shown). Because the latter is a Rhomboid target gene that cell autonomously directs denticle formation (Payre et al. 1999), this explains why the denticle belts do not develop normally in brinker and tsh mutants.

The cuticle phenotype of the brinker tsh double mutants suggests that Brinker and Tsh act together to antagonize Wg signaling in the ventral epidermis. Therefore, the function of Brinker in antagonizing $\mathrm{Wg}$ does not appear to be limited to the embryonic midgut.

\section{Discussion}

Previous analyses have suggested that Brinker may be dedicated to antagonizing Dpp signaling (Campbell and Tomlinson 1999; Jazwinska et al. 1999a; Minami et al. 1999; Ashe et al. 2000; Sivasankaran et al. 2000; Hasson et al. 2001). This appeared particularly likely, given that the Brinker repressor was found to bind to the same sites as the Dpp effector Mad, and thus to compete with activated Mad for binding to Dpp target genes (Kirkpatrick et al. 2001; Rushlow et al. 2001; Saller and Bienz 2001; Zhang et al. 2001).

We have now discovered that Brinker also antagonizes Wg signaling in two embryonic tissues. However, the underlying repressive mechanism appears to be distinct, as the Brinker repressor binds to a site distinct from that occupied by the Wg effector, the dTCF/Armadillo activator complex. Brinker thus acts at short range to block the activity of this complex.

\section{The Brinker/Tsh repressor quenches the activity of dTCF/Armadillo}

Most likely, Brinker uses a mechanism called quenching to block dTCF/Armadillo (Fig. 7). Quenching involves interaction of repressors (and the corepressors they recruit) with activators bound to nearby sites (Gray and Levine 1996). Brinker is known to be able to quench target genes by recruiting the corepressor Groucho (Hasson et al. 2001; Zhang et al. 2001), which is involved in multiple quenching processes (Zhang and Levine 1999). groucho antagonizes wg (Cavallo et al. 1998), and TCF
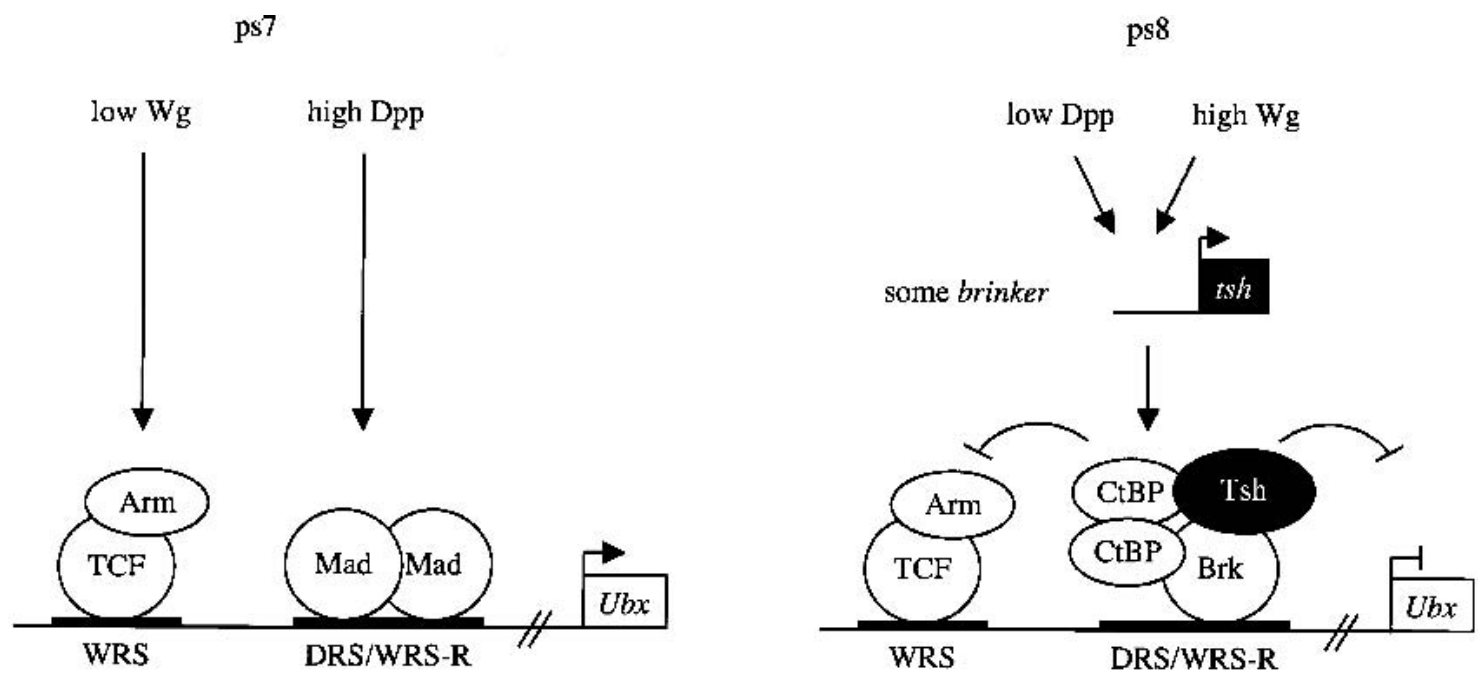

Figure 7. A morphogenetic switch in the $U b x$ midgut enhancer: a model. In ps7, low Wg levels synergize with high Dpp levels to stimulate $U b x$ B; the WRS is occupied by dTCF/Armadillo, the WRS-R by activated Mad. In ps8, the Dpp-signaling levels are low, so Brinker (which is expressed in ps8; Fig. 1A) displaces Mad from the WRS-R. In addition, high Wingless levels (and low Dpp) induce local expression of Tsh in this region. Brinker thus recruits Tsh to the WRS-R, and both proteins together may recruit the quenching factor dCtBP to block the stimulatory activity of the nearby-bound dTCF/Armadillo transcription complex. Note that, although on overexpression, Brinker is sufficient to repress $U b x \mathrm{~B}$ in the absence of $t s h$ (Fig. 5) at normal expression levels, Brinker critically depends on tsh to repress $U b x$ B (Fig. 2). Thus, Tsh is the pivotal factor in this process. Tsh is up-regulated by high Wg levels and, on recruitment by Brinker, confers repression by quenching Wg-mediated activation. 
factors can bind to Groucho proteins directly (Levanon et al. 1998; Roose et al. 1998), so dTCF may thus be able to recruit Groucho unassisted. However, these findings do not rule out the possibility that dTCF relies on cooperation with Brinker to achieve Groucho recruitment.

Brinker can bind to the corepressor dCtBP (Fig. 4), so Brinker may recruit dCtBP instead of, or in addition to, Groucho. Recall that Tsh plays a critical role in the Wgmediated repression in the midgut (Waltzer et al. 2001), just like Brinker itself (Fig. 2). Moreover, Tsh can bind to Brinker as well as to dCtBP, so it seems plausible that Tsh plays a pivotal role in assisting Brinker in the recruitment of dCtBP. Like Groucho, dCtBP is a corepressor with quenching activity (Nibu et al. 1998b). In addition, Tsh may itself be involved in the quenching process. It has been suggested that quenching may be based on obstruction of the interaction between the activation domain of a transcriptional activator and the general transcription machinery (Courey and Jia 2001)-intriguingly, hypophosphorylated Tsh binds to the carboxyterminal activation domain of Armadillo to modulate Wg signaling (Gallet et al. 1999).

Interpretation of distinct Wg-signaling thresholds: a morphogenetic switch at the transcriptional level

The Drosophila midgut has provided a model system in which Wg signaling regulates gene transcription in a concentration-dependent manner; low signaling levels activate $\mathrm{Wg}$ target genes, whereas high levels repress the same genes. The discovery that Brinker confers transcriptional repression by Wg completes our picture of the DNA-binding proteins that interpret these different signaling thresholds. dTCF confers Wg-induced stimulation of target genes (Riese et al. 1997), but its activity can be blocked by Brinker, which confers Wg-mediated repression of the same genes (Fig. 7). dTCF depends on Armadillo for its activity, whereas Brinker depends on Tsh to block the activity of the dTCF/Armadillo complex. In turn, the availability of Armadillo depends directly on Wg signaling, which promotes its stabilization and nuclear translocation (Cavallo et al. 1997), whereas the availability of Tsh depends on transcription of its gene (which itself depends on wg; Mathies et al. 1994). In other words, high Wg signaling induces locally the expression of the Tsh corepressor, which then cooperates with Brinker to repress Wg target genes in the same cells (Fig. 7). One of these targets is wg itself, so Brinker and Tsh take part in the negative feedback loop of Wg signaling in the middle midgut.

\section{The link to Dpp signaling}

$U b x \mathrm{~B}$ is not only a Wg-responsive enhancer, but it is also stimulated by Dpp signaling (Thüringer and Bienz 1993). Furthermore, Dpp signaling antagonizes Wg-mediated repression (Yu et al. 1998). This can be explained in two ways. First, high levels of Dpp-activated Mad are expected to compete with Brinker for binding to the
WRS-R. Second, the brinker gene itself may be downregulated by Dpp signaling, as this is the case in other tissues (Campbell and Tomlinson 1999; Jazwinska et al. 1999a; Minami et al. 1999; Marty et al. 2000; Sivasankaran et al. 2000), so Brinker may only be present at very low levels in cells within the Dpp-signaling domain. We cannot detect brinker expression in this domain, whereas low levels of expression are detectable in the neighboring Wg-signaling domain (in ps8; Fig. 1A). In contrast, in the latter domain, in which the levels of activated Mad are expected to be low, Brinker successfully competes with Mad for binding to the WRS-R and, together with Tsh, which is present at high levels in this domain, blocks the activity of dTCF/Armadillo (Fig. 7). Note that Dpp signaling promotes this repression indirectly, by contributing to the stimulation of Tsh expression in ps8 (Mathies et al. 1994).

Dpp and Wg signaling cooperate in multiple developmental contexts. In some contexts they synergize (e.g., Campbell et al. 1993; Cohen et al. 1993; Thüringer and Bienz 1993), whereas in other contexts, they antagonize each other (e.g., Ma and Moses 1995; Treisman and Rubin 1995; Theisen et al. 1996). Given that most, if not all, Dpp target genes, and multiple Wg target genes, are repressible by Brinker, this suggests that Brinker may have a universal key role in this decision between synergy and antagonism; absence of Brinker allows synergy between Dpp and Wg, whereas presence of Brinker (and Tsh) mediates antagonism.

\section{Materials and methods}

\section{Fly stains}

The loss-of-function alleles brk $^{F 124}$ (Lammel et al. 2000), brk ${ }^{M 68}$ (Jazwinska et al. 1999a), and $t s h^{8}$ (Fasano 1991) were used for analysis. The following lac $Z$ reporter constructs were used: RP9, Ubx B (Thüringer et al. 1993), and BM2 (Szüts et al. 1998). Wg was ubiquitously overexpressed using hs-wg (Noordermeer et al. 1992). The following Gal4 driver and producer lines were used: 24B.Gal4 (for mesodermal expression) (Brand and Perrimon 1993), Arm.Gal4 (for ubiquitous expression) (Sanson et al. 1996), UAS.Wg (Lawrence et al. 1996), UAS.Tsh (Gallet et al. 1998), and UAS.Brk (Lammel et al. 2000).

\section{Heat-shock regimes and phenotypic analyses}

Standard crosses were set up, and embryos were collected at $25^{\circ} \mathrm{C}$. Mutant embryos and embryos overexpressing various proteins were identified by blue balancers and by their gut phenotypes. To generate the brk; tsh double-mutant strain, GFP balancer chromosomes were used that allow unambigous identification of double-mutant larval cuticles.

The following conditions were used to express Wg ubiquitously from the hs-wg transposon: embryos were collected on apple plates for $16 \mathrm{~h}$ at $18^{\circ} \mathrm{C}$, then immersed in a $37^{\circ} \mathrm{C}$ waterbath for $30 \mathrm{~min}$. Subsequently, the embryos were aged for $2 \mathrm{~h}$ at $25^{\circ} \mathrm{C}$ prior to the next heat shock or fixation. Embryos were subjected to three heat shocks. Staining of embryos was performed as described (Yu et al. 1996). The following antibodies were used: anti-lacZ (Promega), anti-Wg (Brook and Cohen 1996).

Cuticle preparations were done as described (Szüts et al. 1997). 


\section{GST fusion proteins, pull-down, and gel-shift assays}

Full-length dCtBP, full-length Brinker, and Brinker fragments were subcloned into pGEX-2TK (Pharmacia) or pT7ßlink using standard PCR-cloning procedures. Full-length Tsh subcloned into pGEX-2T or pT7ßlink was a gift from Lucas Waltzer (Centre de Biologie du Dévelppemont Université Paul Sabatier, Toulouse, France). GST fusion proteins were produced in Escherichia coli BL21, purified by affinity chromatography on GST-agarose beads, and eluted for gel-shift assays as described (Saller and Bienz 2001). Gel-shift experiments with the WRS-R probe were performed as described (Saller and Bienz 2001). For super-shift assays, purified GST-Tsh fusion protein was added to the DNA/ GST-Brinker incubation mix for 10 min prior to loading on the gel. Pull-down assays were performed as described (Waltzer and Bienz 1998).

\section{Acknowledgments}

We thank Lucas Waltzer, Susan Parkhurst, Uwe Lammel, and Stephen Kerridge for plasmids and fly strains, Jan de Boer and Barry Thomson for advice and discussion. E.S. is supported by a long-term fellowship from the Swiss National Science Foundation.

The publication costs of this article were defrayed in part by payment of page charges. This article must therefore be hereby marked "advertisement" in accordance with 18 USC section 1734 solely to indicate this fact.

\section{References}

Ashe, H.L., Mannervik, M., and Levine, M. 2000. Dpp signaling thresholds in the dorsal ectoderm of the Drosophila embryo. Development 127: 3305-3312.

Bienz, M. 1994. Homeotic genes and positional signaling in the Drosophila viscera. Trends Genet. 10: 22-26.

- 1996. Induction of the endoderm in Drosophila. Sem. Cell Dev. Biol. 7: 113-119.

- 1997. Endoderm induction in Drosophila: The nuclear targets of the inducing signals. Curr. Opin. Genet. Dev. 7: 683-688.

. 1998. TCF: Transcriptional activator or repressor? Curr. Opin. Cell. Biol. 10: 366-372.

Bienz, M. and Tremml, G. 1988. Domain of Ultrabithorax expression in Drosophila visceral mesoderm from autoregulation and exclusion. Nature 33: 576-578.

Bienz, M., Saari, G., Tremml, G., Müller, J., Züst, B., and Lawrence, P.A. 1988. Differential regulation of Ultrabithorax in two germ layers of Drosophila. Cell 53: 567-576.

Boyd, J.M., Subramanian, T., Schaeper, U., La Regina, M., Bayley, S., and Chinnadurai, G. 1993. A region in the C-terminus of adenovirus 2/5 E1a protein is required for association with a cellular phosphoprotein and important for the negative modulation of T24-ras mediated transformation, tumorigenesis and metastasis. EMBO J. 12: 469-478.

Brand, A.H. and Perrimon, N. 1993. Targeted gene expression as a means of altering cell fates and generating dominant phenotypes. Development 118: 401-415.

Brook, W.J. and Cohen, S.M. 1996. Antagonistic interactions between wingless and decapentaplegic responsible for dorsal-ventral pattern in the Drosophila leg. Science 273: 13731377.

Brunner, E., Peter, O., Schweizer, L., and Basler, K. 1997. pangolin encodes a Lef-1 homologue that acts downstream of Armadillo to transduce the Wingless signal in Drosophila. $\mathrm{Na}$ ture 385: 829-833.
Cadigan, K.M., Fish, M.P., Rulifson, E.J., and Nusse, R. 1998. Wingless repression of Drosophila frizzled 2 expression shapes the Wingless morphogen gradient in the wing. Cell 93: 767-777.

Campbell, G. and Tomlinson, A. 1999. Transducing the Dpp morphogen gradient in the wing of Drosophila:Regulation of Dpp targets by brinker. Cell 96: 553-562.

Campbell, G., Weaver, T., and Tomlinson, A. 1993. Axis specification in the developing Drosophila appendage: The role of wingless, decapentaplegic, and the homeobox gene aristaless. Cell 74: 1113-1123.

Cavallo, R., Rubenstein, D., and Peifer, M. 1997. Armadillo and dTCF: A marriage made in the nucleus. Curr. Opin. Genet. Dev. 7: 459-466.

Cavallo, R.A., Cox, R.T., Moline, M.M., Roose, J., Polevoy, G.A., Clevers, H., Peifer, M., and Bejsovec, A. 1998. Drosophila Tcf and Groucho interact to repress Wingless signaling activity. Nature 395: 604-608.

Cohen, B., Simcox, A.A., and Cohen, S.M. 1993. Allocation of the thoracic imaginal primordia in the Drosophila embryo. Development 117: 597-608.

Courey, A.J. and Jia, S. 2001. Transcriptional repression: The long and the short of it. Genes \& Dev. 15: 2786-2796.

Criqui-Filipe, P., Ducret, C., Maira, S.M., and Wasylyk, B. 1999. Net, a negative Ras-switchable TCF, contains a second inhibition domain, the CID, that mediates repression through interactions with CtBP and de-acetylation. EMBO $J$. 18: 3392-3403.

Fasano, L., Röder, L., Coré, N., Alexandre, E., Vola, C., Jacq, B., Kerridge, S. 1991. The gene teashirt is required for the development of Drosophila embryonic trunk segments and encodes a protein with widely spaced zinc finger motifs. Cell 64: 63-79.

Gallet, A., Erkner, A., Charroux, B., Fasano, L., and Kerridge, S. 1998. Trunk-specific modulation of wingless signaling in Drosophila by teashirt binding to armadillo. Curr. Biol. 8: 893-902.

Gallet, A., Angelats, C., Erkner, A., Charroux, B., Fasano, L., and Kerridge, S. 1999. The C-terminal domain of armadillo binds to hypophosphorylated teashirt to modulate wingless signaling in Drosophila. EMBO J. 18: 2208-2217.

Gallet, A., Angelats, C., Kerridge, S., and Therond, P.P. 2000. Cubitus interruptus-independent transduction of the Hedgehog signal in Drosophila. Development 127: 5509-5522.

Gray, S. and Levine, M. 1996. Short-range transcriptional repressors mediate both quenching and direct repression within complex loci in Drosophila. Genes \& Dev. 10: 700710 .

Grosschedl, R., Giese, K., and Pagel, J. 1994. HMG domain proteins: Architectural elements in the assembly of nucleoprotein structures. Trends Genet. 10: 94-100.

Hasson, P., Muller, B., Basler, K., and Paroush, Z. 2001. Brinker requires two corepressors for maximal and versatile repression in Dpp signaling. EMBO J. 20: 5725-5736.

Hooper, J.E. 1994. Distinct pathways for autocrine and paracrine Wingless signaling in Drosophila embryos. Nature 372: 461-464.

Hoppler, S. and Bienz, M. 1995. Two different thresholds of wingless signaling with distinct developmental consequences in the Drosophila midgut. EMBO J. 14: 5016-5026.

Jazwinska, A., Kirov, N., Wieschaus, E., Roth, S., andRushlow, C. 1999a. The Drosophila gene brinker reveals a novel mechanism of Dpp target gene regulation. Cell 96: 563-573.

Jazwinska, A., Rushlow, C., and Roth, S. 1999b. The role of brinker in mediating the graded response to Dpp in early Drosophila embryos. Development 126: 3323-3334. 
Jiang, J. and Struhl, G. 1996. Complementary and mutually exclusive activities of decapentaplegic and wingless organize axial patterning during Drosophila leg development. Cell 86: 401-409.

Kim, J., Johnson, K., Chen, H.J., Carroll, S., and Laughon, A. 1997. Drosophila Mad binds to DNA and directly mediates activation of vestigial by Decapentaplegic. Nature 388: 304 308.

Kirkpatrick, H., Johnson, K., and Laughon, A. 2001. Repression of dpp targets by binding of brinker to mad sites. J. Biol. Chem. 276: 18216-18222.

Klein, T. 2001. Wing disc development in the fly: The early stages. Curr. Opin. Genet. Dev. 11: 470-475.

Lammel, U., Meadows, L., and Saumweber, H. 2000. Analysis of Drosophila salivary gland, epidermis and CNS development suggests an additional function of brinker in anterior-posterior cell fate specification. Mech. Dev. 92: 179-191.

Lawrence, P.A., Sanson, B., and Vincent, J.P. 1996. Compartments, wingless and engrailed: Patterning the ventral epidermis of Drosophila embryos. Development 122: 4095-4103.

Lecuit, T. and Cohen, S.M. 1997. Proximal-distal axis formation in the Drosophila leg. Nature 388: 139-145.

Levanon, D., Goldstein, R.E., Bernstein, Y., Tang, H., Goldenberg, D., Stifani, S., Paroush, Z., and Groner, Y. 1998. Transcriptional repression by AML1 and LEF-1 is mediated by the TLE/Groucho corepressors. Proc. Natl. Acad. Sci. 95: 1159011595.

Ma, C. and Moses, K. 1995. Wingless and patched are negative regulators of the morphogenetic furrow and can affect tissue polarity in the developing Drosophila compound eye. Development 121: 2279-2289.

Marty, T., Muller, B., Basler, K., and Affolter, M. 2000. Schnurri mediates dpp-dependent repression of brinker transcription. Nat. Cell. Biol. 2: 745-749.

Mathies, L.D., Kerridge, S., and Scott, M.P. 1994. Role of the teashirt gene in Drosophila midgut morphogenesis: Secreted proteins mediate the action of homeotic genes. Development 120: 2799-2809.

Minami, M., Kinoshita, N., Kamoshida, Y., Tanimoto, H., and Tabata, T. 1999. brinker is a target of Dpp in Drosophila that negatively regulates Dpp-dependent genes. Nature 398: 242 246.

Neumann, C.J. and Cohen, S.M. 1997. Long-range action of Wingless organizes the dorsal-ventral axis of the Drosophila wing. Development 124: 871-880.

Nibu, Y. and Levine, M.S. 2001. CtBP-dependent activities of the short-range Giant repressor in the Drosophila embryo. Proc. Natl. Acad. Sci. 98: 6204-6208.

Nibu, Y., Zhang, H., Bajor, E., Barolo, S., Small, S., and Levine, M. 1998a. dCtBP mediates transcriptional repression by Knirps, Krüppel and Snail in the Drosophila embryo. EMBO J. 17: 7009-7020.

Nibu, Y., Zhang, H., and Levine, M. 1998b. Interaction of shortrange repressors with Drosophila CtBP in the embryo. Science 280: 101-104.

Noordermeer, J., Johnston, P., Rijsewijk, F., Nusse, R., and Lawrence, P.A. 1992. The consequences of ubiquitous expression of the wingless gene in the Drosophila embryo. Development 116: 711-719.

O'Keefe, L., Dougan, S.T., Gabay, L., Raz, E., Shilo, B.Z., and DiNardo, S. 1997. Spitz and Wingless, emanating from distinct borders, cooperate to establish cell fate across the Engrailed domain in the Drosophila epidermis. Development 124: 4837-4845.

Payre, F., Vincent, A., and Carreno, S. 1999. ovo/svb integrates Wingless and DER pathways to control epidermis differen- tiation. Nature 400: 271-275.

Perrimon, N. 1994. The genetic basis of patterned baldness in Drosophila. Cell 76: 781-784.

Phippen, T.M., Sweigart, A.L., Moniwa, M., Krumm, A., Davie, J.R., and Parkhurst, S.M. 2000. Drosophila C-terminal binding protein functions as a context-dependent transcriptional co-factor and interferes with both mad and groucho transcriptional repression. J. Biol. Chem. 275: 37628-37637.

Piepenburg, O., Vorbruggen, G., and Jäckle, H. 2000. Drosophila segment borders result from unilateral repression of hedgehog activity by wingless signaling. Mol. Cell 6: 203-209.

Poortinga, G., Watanabe, M., and Parkhurst, S.M. 1998. Drosophila CtBP: A Hairy-interacting protein required for embryonic segmentation and hairy-mediated transcriptional repression. EMBO J. 17: 2067-2078.

Riese, J., Yu, X., Munnerlyn, A., Eresh, S., Hsu, S.C., Grosschedl, R., and Bienz, M. 1997. LEF-1, a nuclear factor coordinating signaling inputs from wingless and decapentaplegic. Cell 88: 777-787.

Roose, J., Molenaar, M., Peterson, J., Hurenkamp, J., Brantjes, H., Moerer, P., van de Wetering, M., Destree, O., and Clevers, H. 1998. The Xenopus Wnt effector XTcf-3 interacts with Groucho-related transcriptional repressors. Nature 395: 608-612.

Royet, J. and Finkelstein, R. 1996. hedgehog, wingless and orthodenticle specify adult head development in Drosophila. Development 122: 1849-1858.

Rulifson, E.J., Micchelli, C.A., Axelrod, J.D., Perrimon, N., and Blair, S.S. 1996. wingless refines its own expression domain on the Drosophila wing margin. Nature 384: 72-74.

Rushlow, C., Colosimo, P.F., Lin, M.C., Xu, M., and Kirov, N. 2001. Transcriptional regulation of the Drosophila gene zen by competing Smad and Brinker inputs. Genes \& Dev. 15: 340-351.

Saller, E. and Bienz, M. 2001. Direct competition between Brinker and Drosophila Mad in Dpp target gene transcription. EMBO Rep. 2: 298-305.

Sanson, B., White, P., and Vincent, J.P. 1996. Uncoupling cadherin-based adhesion from wingless signaling in Drosophila. Nature 383: 627-630.

Sivasankaran, R., Vigano, M.A., Muller, B., Affolter, M., and Basler, K. 2000. Direct transcriptional control of the Dpp target omb by the DNA binding protein Brinker. EMBO $J$. 19: 6162-6172.

Struhl, G. and Basler, K. 1993. Organizing activity of wingless protein in Drosophila. Cell 72: 527-540.

Szüts, D., Freeman, M., and Bienz, M. 1997. Antagonism between EGFR and Wingless signaling in the larval cuticle of Drosophila. Development 124: 3209-3219.

Szüts, D., Eresh, S., and Bienz, M. 1998. Functional intertwining of Dpp and EGFR signaling during Drosophila endoderm induction. Genes \& Dev. 12: 2022-2035.

Theisen, H., Haerry, T.E., O'Connor, M.B., and Marsh, J.L. 1996. Developmental territories created by mutual antagonism between Wingless and Decapentaplegic. Development 122: 3939-3948.

Thüringer, F. and Bienz, M. 1993. Indirect autoregulation of a homeotic Drosophila gene mediated by extracellular signaling. Proc. Nat. Acad. Sci. 90: 3899-3903.

Thüringer, F., Cohen, S.M., and Bienz, M. 1993. Dissection of an indirect autoregulatory response of a homeotic Drosophila gene. $E M B O$ I. 12: 2419-30.

Treisman, J.E. and Rubin, G.M. 1995. wingless inhibits morphogenetic furrow movement in the Drosophila eye disc. Development 121: 3519-3527.

Turner, J. and Crossley, M. 1998. Cloning and characterization 
Saller et al.

of mCtBP2, a co-repressor that associates with basic Krüppel-like factor and other mammalian transcriptional regulators. EMBO J. 17: 5129-5140.

van de Wetering, M., Cavallo, R., Dooijes, D., van Beest, M., van Es, J., Loureiro, J., Ypma, A., Hursh, D., Jones, T., Bejsovec, A., et al. 1997. Armadillo coactivates transcription driven by the product of the Drosophila segment polarity gene dTCF. Cell 88: 789-799.

Waltzer, L. and Bienz, M. 1998. Drosophila CBP represses the transcription factor TCF to antagonize Wingless signaling. Nature 395: 521-525.

Waltzer, L., Vandel, L., and Bienz, M. 2001. Teashirt is required for transcriptional repression mediated by high Wingless levels. EMBO J. 20: 137-145.

Yang, X., van Beest, M., Clevers, H., Jones, T., Hursh, D.A., and Mortin, M.A. 2000. decapentaplegic is a direct target of dTcf repression in the Drosophila visceral mesoderm. Development 127: 3695-3702.

Yu, X., Hoppler, S., Eresh, S., andBienz, M. 1996. decapentaplegic, a target gene of the wingless signaling pathway in the Drosophila midgut. Development 122: 849-858.

Yu, X., Riese, J., Eresh, S., and Bienz, M. 1998. Transcriptional repression due to high levels of Wingless signaling. EMBO $J$. 17: 7021-7032.

Zecca, M., Basler, K., and Struhl, G. 1996. Direct and long-range action of a wingless morphogen gradient. Cell 87: 833-844.

Zhang, H. and Levine, M. 1999. Groucho and dCtBP mediate separate pathways of transcriptional repression in the Drosophila embryo. Proc. Natl. Acad. Sci. 96: 535-540.

Zhang, H., Levine, M., and Ashe, H.L. 2001. Brinker is a sequence-specific transcriptional repressor in the Drosophila embryo. Genes \& Dev. 15: 261-266. 


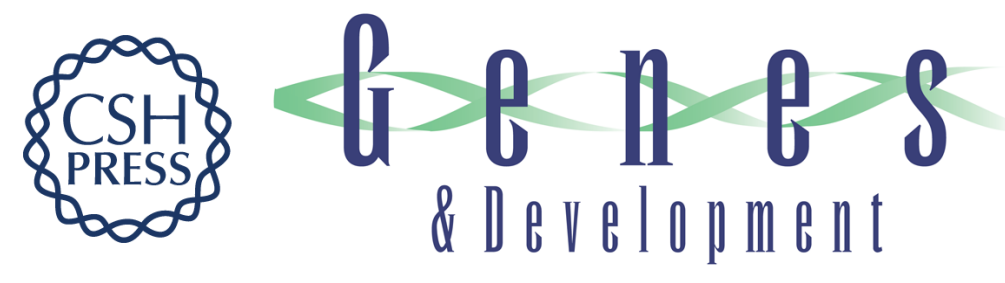

\section{The transcriptional repressor Brinker antagonizes Wingless signaling}

Elisabeth Saller, Ann Kelley and Mariann Bienz

Genes Dev. 2002, 16:

Access the most recent version at doi:10.1101/gad.230002

References This article cites 77 articles, 39 of which can be accessed free at: http://genesdev.cshlp.org/content/16/14/1828.full.html\#ref-list-1

License

Email Alerting Receive free email alerts when new articles cite this article - sign up in the box at the top Service right corner of the article or click here.

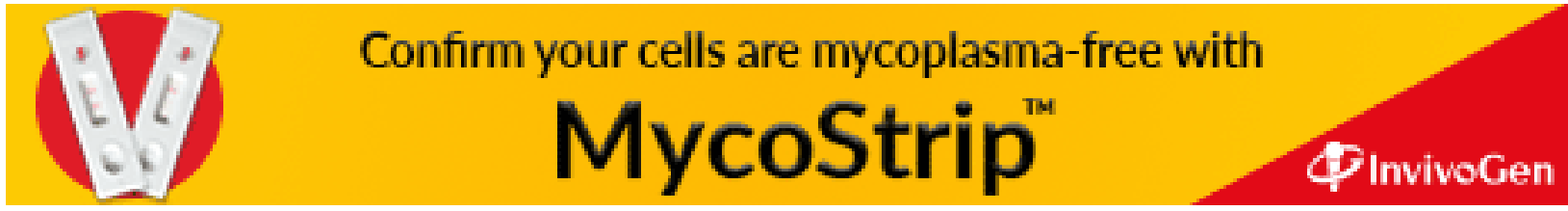

